\title{
Oil-in-Water Nanocontainers as Low Environmental Impact Cleaning Tools for Works of Art: Two Case Studies
}

\author{
Emiliano Carretti, Rodorico Giorgi, Debora Berti, and Piero Baglioni* \\ Department of Chemistry and CSGI, University of Florence, via della Lastruccia 3 - Sesto Fiorentino,
} 50019 Florence, Italy

Received February 19, 2007. In Final Form: March 14, 2007

\begin{abstract}
A novel class of $p$-xylene-in-water microemulsions mainly based on nonionic surfactants and their application as low impact cleaning tool in cultural heritage conservation is presented. Alkyl polyglycosides (APG) and Triton X-100 surfactants allow obtaining very effective low impact oil-in-water $(\mathrm{o} / \mathrm{w})$ microemulsions as alternatives to pure organic solvents for the removal of polymers (particularly Paraloid B72 and Primal AC33) applied during previous conservation treatments. The ternary APG/ $p$-xylene/water microemulsions have been characterized by quasi elastic light scattering to obtain the hydrodynamic radius and the polydispersity of the microemulsion droplets. Laplace inversion of the correlation function CONTIN analysis provided evidence of acrylic copolymers solubilization into the oil nanodroplets. Contact angle, Fourier transform infrared (FTIR), and scanning electron microscopy/energy-dispersive spectroscopy (SEM/EDS) data confirmed that microemulsions were effective in removing polymer coatings. The phase diagram of APG microemulsions showed that a reduction $>90 \%$ (compared to the conventional cleaning methods) of the organic solvent can be achieved by using o/w microemulsions. The microemulsions were successfully tested in two real cases: (1) the APG based microemulsion was used in a Renaissance painting by Vecchietta in Santa Maria della Scala, Siena, Italy, degraded by the presence of a polyacrylate coating applied during a previous restoration and (2) a Triton X-100 oil-in-water microemulsion containing $\left(\mathrm{NH}_{4}\right)_{2} \mathrm{CO}_{3}$ in the water continuous phase. The association of ammoniun carbonate to the microemusion led to the swelling of an organic deposit (mainly asphaltenes deposited on the fresco in the Oratorio di San Nicola al Ceppo in Florence, still contamined by the water of the Arno river during the 1966 flood) and a very efficient removal of highly insoluble inorganic deposits (mainly gypsum) strongly associated to asphaltenes. These innovative systems are very attractive for the low amount of organic solvent used to extract the polymers or highly insoluble substances as the asphaltene and the very efficient and mild impact of the cleaning procedure on the fragile painted surfaces.
\end{abstract}

\section{Introduction}

The intervention for the preservation and restoration of cultural heritage often implies the simultaneous solutions of multiple issues as the preservation and the consolidation of the original materials and the selective removal of all the substances not appertaining to the work of art, usually applied during a previous restoration. The cleaning of the surface of a painting is one of the most important and delicate operations because it can be, when improperly performed, aggressive and invasive for the original materials. During the past years, $\mathrm{we}^{1,2}$ developed new cleaning procedures for works of art that we mainly applied to wall paintings contaminated by accidental or natural deposition of pollutants or by the wrong application of polymeric materials, mainly acrylic and vinyl polymers and their derivatives largely, but often improperly, used in the restorations for the consolidation of pictorial layers. In both cases, the main problem is related to the aging of the organic substances that, at the same time, reduces their solubility in organic solvents, causes an alteration of their color, as shown as an example in Figure 1, and catalyzes the degradation of the painted layer. ${ }^{3-7}$

* To whom correspondence should be addressed. E-mail: baglioni@ csgi.unifi.it.

(1) Carretti, E.; Dei, L.; Baglioni, P. Langmuir 2003, 19, 7867.

(2) Carretti, E.; Dei, L.; Baglioni, P. Stud. Conserv. 2005, 50, 1.

(3) Ciabach, J. Proceedings of the Symposium 'Resins in Conservation', Edimburgh, UK, May 21-22, 1982; pp 5.1-5.8.

(4) Luskin, L. S. In Vynil and Diene Monomers; Leonard, E. C., Ed.; WileyInterscience: New York, 1980; Part I, pp 105-204.

(5) Horie, C. V. Materials for Conservation; Butterworth: London, 1987.

(6) Tintori, L. Stud. Conserv. 1963, 8, 37.

(7) Mora, P.; Mora Sbordoni, L. In Art and Eternity: the Nefertari Wall Paintings Conservation Project; Corzo, M. A., Afshar, M., Ed.; Getty Conservation Institute: Los Angeles, CA, 1993; pp 67-81.
Figure 1A reports a particular of a 16th century fresco in the San Salvador Church in Venice treated 40 years ago with a thick layer of poly-EMA/MA (paraloid) degraded during the years and still present as coating on the surface of the painting. The aging of the paraloid, besides the degradation of the painted layer, produced a strong change of the polychromy because of the bathocromic shift of the UV band (Figure 1B) of the polymer during the aging. As a consequence, all the colors, especially the background, appear darker and the coated surface is very shiny. Another example is reported in Figure 2 that shows the surface of the wall paintings in the Oratorio di San Nicola al Ceppo in Florence, Italy ( 17 th century) affected by a thick layer of insoluble black crusts constituted by a mixture of $\mathrm{CaSO}_{4} \cdot 2 \mathrm{H}_{2} \mathrm{O}$ and asphaltenes (inlet) originated by the deposition of the insoluble fraction of mineral combustibles during the Arno's flood in $1966 .{ }^{8}$ Considering that these crusts were insoluble to pure solvents for the removal of the organic fraction, making impossible the successive extraction of inorganic contaminants, we formulated a new procedure to simultaneously remove highly insoluble organic and inorganic materials. This new method minimizes the invasiveness of the treatment, without releasing side products within the restored porous structure.

Our research group has pioneered the use of microemulsions in cultural heritage conservation ${ }^{1,2}$ as solubilizing agents to be used as an alternative to pure organic solvents for the selective extraction of naturally or artificially aged polymeric coatings. Nanotechnologies have produced some meaningful advantages within this field reducing between $80 \%$ and $95 \%$ of the amount

(8) Pintus, S.; Messeri, S. 4th November 1966. The flood in Florence; Ibiskos Editrice: Firenze, Italy, 2006. 

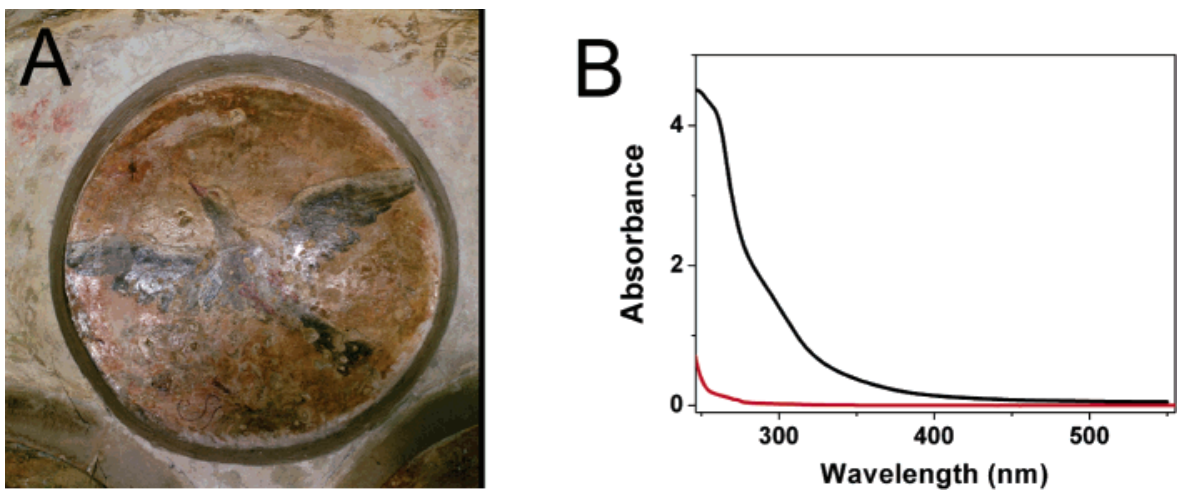

Figure 1. Particular of a fresco in the Sacristy of the San Salvador church, Venice, Italy. (A) Image of a particular of the fresco interested by the presence of a 40 year naturally aged EMA/MA 70:30 based coating. (B) UV spectra of the aged (black line) and fresh (red line) EMA/MA 70:30.

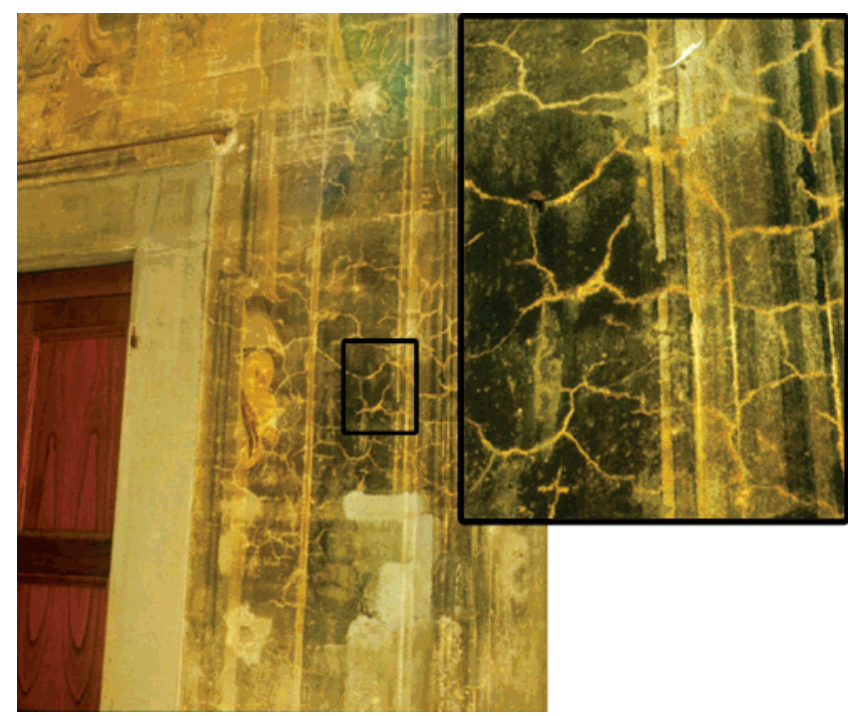

Figure 2. Particular of the wall paintings in the Oratorio di San Nicola al Ceppo, Florence, Italy (17th century) still affected (2006) by a surface layer of black crusts composed of asphaltenes and gypsum originated by the flood of Arno river in 1966. In the inlet is reported an enlargment highlighting the presence of black crusts.

of the used organic solvents with a consequent reduction of the environmental impact. ${ }^{1}$ Frescoes are porous structures, and conventional solvents can be efficient in removing polymeric material at the surface but are almost completely inefficient in cleaning the porous structure. Nanocompartmentalized systems are the best available cleaning system to avoid the penetration and the diffusion of the removed polymeric materials into the porous structure of the work of art.

In this paper, we present significant improvements of this method developing new oil-in-water microemulsions. As oil, we used $p$-xylene for the removal of organic materials from painted surfaces as it is very efficient in solubilizing acrylic polymers. We formulated a nonionic (Triton X-100) or mixed ionic/nonionic (alkyl polyglycosides, APG) p-xylene-in-water (o/w) microemulsions that are effective in the solubilization of naturally aged hydrophobic materials. Attention was focused on nonionic surfactants because they have a very low critical micellar concentration allowing a reduction of the total amount of the nonvolatile organic phase (surfactant).

Microemulsion systems were tailored for two specific applications that are reported in this study: (1) a microemulsion containing a very low amount of organic solvent specifically formulated for paraloid removal from "a secco" paintings (the cleaning of a "a secco" paintings is very difficult with conventional
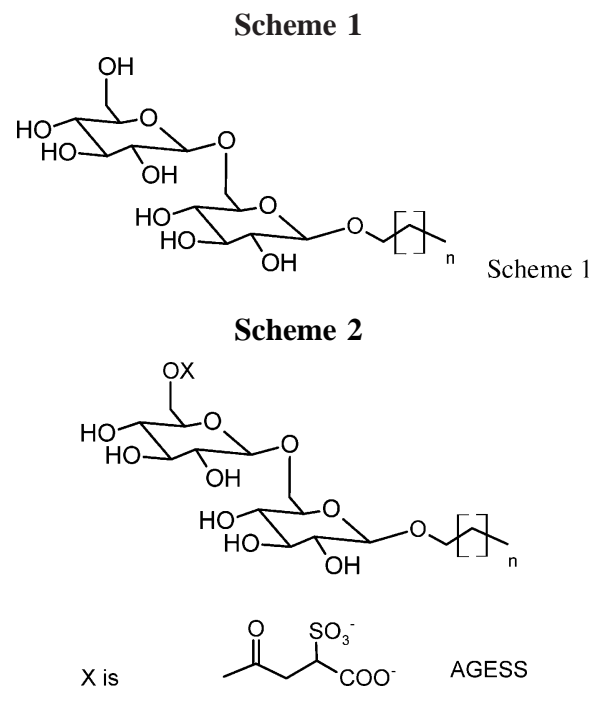

methods) and (2) a micromulsion containing ammonium carbonate to remove 40 -year-old asphaltene-calcium sulfate crusts.

\section{Experimental Section}

Materials. $p$-Xylene (purity $>99.5 \%$ ) and ammonium carbonate were purchased from Merck, Darmstadt, Germany; Triton X-100 was purchased from Aldrich, Germany. Nonionic alkyl polyglycoside (AGE, purity > 98.5\%, molecular weight $568.4 \mathrm{~g} / \mathrm{mol}$ ) was synthesized by Lamberti Chemicals Italy. Synthesis of commercial AGE was performed by Fisher glycosidation of a mixture of C10C14 fatty alcohols. ${ }^{9}$ As indicated in the literature, ${ }^{10-12}$ the AGE is obtained under acidic catalysis through a direct reaction between technical grade fatty alcohols and anhydrous glucose, where the excess of alcohols constitutes the reaction medium. The water produced in the reaction was collected in a cooling trap. In that way, as indicated by the producer, the average polymerization degree was 1.2, meaning that, in the average, out of every 100 surfactant molecules 20 have a polar head composed by two sugar units. The structure of the dimer is indicated in Scheme 1.

Sodium alkyl polyglycosides sulfosuccinate (AGESS, purity $>$ $98.5 \%$, calculated molecular weight $879.6 \mathrm{~g} / \mathrm{mol}$ ) was then obtained by means of a reaction of the AGE maleic ester with sodium sulfite according to ref $14^{13}$ and was purified by recrystallization from ethanol. The structure of the obtained surfactant is indicated in Scheme 2 for the dimer molecule.

(9) Fischer, E. Ber. Dtsch. Chem. Ges. 1895, 28, 1145

(10) Böge, K.; Tietze, L. F. Eur. J. Lipid Sci. Technol. 1998, 100, 36.

(11) Lüders, H. In Nonionic surfactants. Alkyl Polyglucosides; Lüders, H., Balzer, D., Eds.; Dekker: New York, 2000; pp 7-75.

(12) Stradner, A.; Mayer, B.; Sottmann, T.; Hermetter, A.; Glatter, O. J. Phys. Chem. B 1999, 103, 6680 .

(13) Garlisi, S.; Fornara, D.; Bernardi, P. European Patent EP0510565, 1992. 
Table 1. Composition (w/w \%) of Microemulsion A and Microemulsion B

\begin{tabular}{|c|c|c|c|c|c|}
\hline $\begin{array}{l}\text { micellar } \\
\text { solution A }\end{array}$ & $\begin{array}{c}\text { composition } \\
\text { (w/w \%) }\end{array}$ & $\begin{array}{c}\text { microemulsion } \\
\text { A }\end{array}$ & $\begin{array}{c}\text { composition } \\
(\mathrm{w} / \mathrm{w} \%)\end{array}$ & $\begin{array}{l}\text { microemulsion } \\
\text { B }\end{array}$ & $\begin{array}{c}\text { composition } \\
(\mathrm{w} / \mathrm{w} \%)\end{array}$ \\
\hline AGE & 0.52 & AGE & 0.52 & Triton X-100 & 4.9 \\
\hline AGESS & 0.12 & AGESS & 0.12 & $\left(\mathrm{NH}_{4}\right)_{2} \mathrm{CO}_{3}$ & 2.1 \\
\hline $\mathrm{H}_{2} \mathrm{O}$ & 99.36 & $\mathrm{H}_{2} \mathrm{O}$ & 99.00 & $\mathrm{H}_{2} \mathrm{O}$ & 92.6 \\
\hline & & $p$-xylene & 0.36 & $p$-xylene & 0.4 \\
\hline
\end{tabular}

All the chemicals were used as received without any further purification.

The microemulsion systems were prepared by using a mixture between AGE-AGESS whose composition is specified in Table 1; for all the surfactants, the alkyl chain is constituted by a distribution of chains with $n$ values equal to 8 (30\%), 10 (60\%), and $12(10 \%)$.

Poly-ethylmethacrylate (EMA)/methylacrylate (MA) copolymer (PolyEMA/MA) with EMA/MA mole ratio 70:30 and average molecular weight 80000 in pellets was obtained from Zecchi, Florence, Italy. Water was purified by a Millipore MilliRO-6 plus MilliQ (Organex System) apparatus: the resistance of water was $>$ $18 \mathrm{M} \Omega . \mathrm{cm}$.

Sample Preparation. Two different o/w microemulsions (Table 1) were prepared by mixing the appropriate components according to the following procedure. The surfactant mixture (micellar solution A) was dissolved in water under stirring until a transparent solution was obtained. The oil phase ( $p$-xylene) was finally added dropwise at room temperature until a stable system was formed; this $\mathrm{o} / \mathrm{w}$ microemulsion was stable for more than 6 months. For microemulsion $\mathrm{B}, p$-xylene was added to the ternary water/Triton $\mathrm{X}-100 /\left(\mathrm{NH}_{4}\right)_{2}-$ $\mathrm{CO}_{3}$ system.

Dynamic Quasi-Elastic Light Scattering (QELS). QELS measurements were carried out with a Brookhaven apparatus (BI200SM with BI9000AT correlator) using the doubled frequency of a Nd:YAG $(532 \mathrm{~nm})$ diode pumped laser with a power attenuated to $20 \mathrm{~mW}$ to avoid sample heating; power stability was $\pm 0.5 \%$. Scattered light was collected by a Thorn-Emi 96350 photomultiplier. Size distribution of the scattering units was obtained by analyzing the autocorrelation function with the constrained regularization $(\text { CONTIN })^{14}$ algorithm and cumulants ${ }^{15}$ methods.

Equilibrium Surface Tension. Critical micelle concentration (cmc) values were obtained from equilibrium surface tension measurements with Du Nouy platinum ring method using a KSV Sigma 70 digital tensiometer (accuracy $0.1 \mathrm{mN} / \mathrm{m}$ ). The measurements were performed at $25 \pm 0.1{ }^{\circ} \mathrm{C}$ by dilution of an aqueous micellar solution of pure APG or AGE/AGESS mixture. The solution was stirred for $5 \mathrm{~min}$ and was allowed to rest $10 \mathrm{~min}$ before each measurement. The final curve was obtained as the result of seven measurements, and the data reported are the average values with corresponding standard deviation. Critical micelle concentration $(\mathrm{cmc})$ values for pure AGE and AGESS are $3.0 \times 10^{-3}$ weight percent $(\mathrm{w} / \mathrm{w} \%)^{16}$ and $1.6 \times 10^{-2} \mathrm{w} / \mathrm{w} \%$, respectively.

Miscibility Gap. The miscibility gap was obtained by visual inspection by placing the surfactant solution in glass tubes into a temperature-controlled bath; the temperature was increased at the rate of $0.1^{\circ} \mathrm{C} / \mathrm{min}$ near the phase-separation temperature.

Contact Angle Measurements. Contact angle measurements ${ }^{17,18}$ were performed on glass and mortar specimens by means of a PC-controlled NRLRamé-Hart Inc. apparatus interfaced to a PC. Water droplets $(5 \mu \mathrm{L})$ were deposited onto the solid surface using a Hamilton microsyringe, and the contact angle was determined $5 \mathrm{~s}$ after the deposition. The contact angles are reported with their standard deviations as the average of 10 measurements. Mortar samples were prepared according to a generally accepted

(14) Provencher, S. W. Comput. Phys. Commun. 1982, 27, 213.

(15) Koppel, D. E. J. Chem. Phys. 1972, 57, 4814

(16) Platz, G.; Polike, J.; Thunig, T.; Hoffmann, R.; Nickel, D.; von Rybinski, W. Langmuir 1995, 11, 4250 .

(17) Drelich, J.; Wilbur, J. L.; Miller, J. D.; Whitesides, G. M. Langmuir 1996, 12,1913

(18) Drelich, J.; Miller, J. D.; Kumar, A.; Whitesides, G. M. Colloids Surf., A: Physicochem. Eng. Aspects 1994, 93, 1. procedure. ${ }^{19}$ The deposition of the poly(EMA/MA) layer onto both glass and mortar surfaces was realized by spreading with a brush a copolymer solution in $p$-xylene $(4 \% \mathrm{w} / \mathrm{w})$; all the measurements were carried out when complete drying of the copolymer film was achieved. The final thickness of the poly(EMA/MA) coating was about $20 \mu \mathrm{m}$.

Scanning Electron Microscopy (SEM). SEM images were collected by means of a Stereoscan S360 instrument, Cambridge (U.K.), after graphitization of the samples. $25 \mathrm{KV}$ (Kilo-Volts) acceleration potential and a $25 \mathrm{~mm}$ as working distance were used. The energy-dispersive spectroscopy (EDS) spectra were recorded using the microprobe $\mathrm{X}$-sight Oxford-Cambridge coupled with the SEM microscope.

Fourier Transform Infrared (FTIR). FTIR spectra were obtained in the microreflectance mode using a BioRad FTS-40 spectrometer equipped with a BioRad UMA500 microscope (MCT detector) with $8 \mathrm{~cm}^{-1}$ resolution and 512 scans.

Application of Microemulsions. The application of microemulsions in some regions of the wall painting by Vecchietta (16th century) and onto the wall paintings into the Oratorio di San Nicola al Ceppo (17th century) was carried out with the "compress technique" 20 with a contact time of almost $2 \mathrm{~h}$ by using wood cellulose pulp to prepare the compresses and by protecting the painted surface by Japanese paper sheets. After removal of the compress, the paint surface was washed with water to completely eliminate all the surfactants' residues (the only nonvolatile component of the microemulsion).

\section{Results}

(1) Characterization and Application of the "Green" APG Microemulsions. Alkyl polyglycosides (APG), because of their favorable physicochemical and environmental properties, are among the surfactants that merit the investigation for a possible application to the field of Cultural Heritage Conservation. Although APG have been known since the end of the 19th century, an economically advantageous way to produce them was discovered only at the end of the 20th century. ${ }^{21}$ Because of their excellent biodegradability, good surface active properties, and being synthesized from renewable biocompatible raw materials, such as sugars and fatty alcohols, alkyl polyglycosides offer many advantages from the economical and ecological point of view. ${ }^{22,23}$ Chemically, alkyl polyglycosides are polymeric acetals of fatty alcohols and glucose, with an average number of glucose molecules in the polar head less than two. Each molecule $\left(\mathrm{C}_{m} \mathrm{G}_{n}\right)$ contains $m$ carbons in the hydrophobic alkyl chain and $n$ glucose units in the hydrophilic headgroup. Research about this class of surfactants has been mainly focused on the study of the phase diagrams of $\mathrm{APG}-\mathrm{H}_{2} \mathrm{O}$ mixtures ${ }^{24,25}$ and on their interfacial and structural ${ }^{26-29}$ or mechanical properties. ${ }^{30}$ Although the interest for this class of surfactant is growing up, the literature on the

(19) Giorgi, R.; Dei, L.; Baglioni, P. Stud. Conserv. 2000, 45, 154

(20) Ferroni, E. In Ecological Physical Chemistry; Rossi, C., Tiezzi, E., Eds.; Elsevier: Amsterdam, 1991; pp 345-358.

(21) Lüders, H.; Balzer, D. In Proceedings of the 2nd World Surfactant Congress, Paris, May 25-28; ASPA Paris Publishers: Paris, 1988; pp 80-93.

(22) Knaut, J.; Kreienfeld, G. G. Riv. Ita. Sostanze Grasse 1994, 71, 307-311.

(23) Schmidt K.; Nieendick, C. In Proceedings of the 6th CID days congress, Rome, October 18-20, 1995; pp 67-84

(24) Balzer, D. Langmuir 1993, 9, 3375.

(25) Hoffmann, B.; Platz, G. Curr. Opin. Colloid Interface Sci. 2001, 6, 171. 


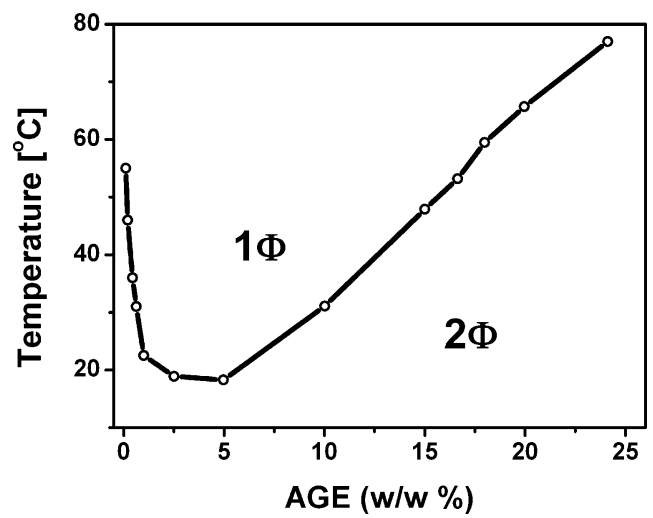

Figure 3. Phase diagram for $\mathrm{AGE}$ in $\mathrm{H}_{2} \mathrm{O}$.

APG microemulsions is still scarce. ${ }^{31-34}$ In this paper, a new APG $p$-xylene-in-water microemulsion obtained from a binary mixture (ionic/nonionic) of two different APGs is presented. Its potential application as cleaning material was tested, in terms of solubilization of poly-ethylmethacrylate/methylacrylate (poly(EMA/MA), Paraloid B72, through QELS and rheology. The efficacy of this formulation was tested during restoration of wall paintings by Vecchietta (15th century) in the Old Sacristy of the Spedale di Santa Maria della Scala in Siena, Italy. Considering that all these paintings have been realized with the a secco technique, the cleaning step is particularly critical because of the fragility of the support. Moreover, the application of pure organic solvents must be avoided to control their penetration, via capillary suction, into the original paint layers that can lead to swelling and leaching of all the water-insoluble organic components (i.e., binders) constituting the paint, from the work of art surface. For all these reasons, the o/w microemulsions presented in this paper, containing more than $95 \% \mathrm{w} / \mathrm{w}$ of water, are the most suitable, effective, and less invasive tool for the selective extraction of the Paraloid B72 surface film without any undesirable side effect.

Since our main target was the setup of a new family of low impact $p$-xylene-in-water microemulsions effective in the solubilization of acrylic copolymers from painted surfaces, we first investigated the $\mathrm{AGE} / \mathrm{H}_{2} \mathrm{O}$ system. Unfortunately, as it is already described for similar $\mathrm{APG}^{24}$ and also indicated in Figure 3, AGE shows in $\mathrm{H}_{2} \mathrm{O}$ a pronounced miscibility gap at the concentrations needed for applicative purposes (between 0.5 and $5 \mathrm{w} / \mathrm{w} \%$ ) in a temperature range between $20{ }^{\circ} \mathrm{C}$ and $40{ }^{\circ} \mathrm{C}$. That makes $p$-xylene $/ \mathrm{H}_{2} \mathrm{O} / \mathrm{AGE}$ microemulsions unsuitable for applicative purposes.

Therefore, the attention was focused on the AGE/AGESS 80: $20 \mathrm{w} / \mathrm{w}$, mixed nonionic-ionic surfactants for which no miscibility gap has been observed, seeking a compromise between proper $p$-xylene incorporation and AGESS percentage in the surfactant mixture (which increases the $\mathrm{cmc}$ and consequently the amount of the nonvolatile components that can be loaded

(26) Nickel, D.; Nitsch, C.; Kurzendorfer, P.; Von Rybinski, W. Prog. Colloid Polym. Sci. 1992, 89, 149-252.

(27) Stradner, A.; Glatter, O.; Schurtenberger, P. Langmuir 2000, 16, 5354.

(28) Johansson, I.; Strandberg, C.; Karlsson, B.; Gustavsson, B. In Proceedings of the 4th World Surfactant Congress-Cesio 1996, Barcelona, June 3-7, 1996; de Llúria, R., Ed.; pp 283-298.

(29) Ericson, C. A.; Söderman, O.; Garamus, V. M.; Bergström, M.; Ulvenlund, S. Langmuir $\mathbf{2 0 0 5}, 21,1507$

(30) Schulte, J.; Enders, S.; Quitzsch, K. Colloid Polym. Sci. 1999, 277, 827.

(31) Ryan, L. D.; Kaler, E. W. Langmuir 1999, 15, 92.

(32) Kahlweit, M.; Busse, G.; Faulhaber, B. Langmuir 1996, 12, 861.

(33) Chai, J. L.; Li, G. Z.; Zhang, G. Y.; Lu, J. J.; Wang, Z. N. Colloids Surf., A: Physicochem. Eng. Aspects 2003, 231, 173.

(34) Fukuda, K.; Soderman, O.; Lindman, B.; Shinoda, K. Langmuir 1993, 9 , 2921.

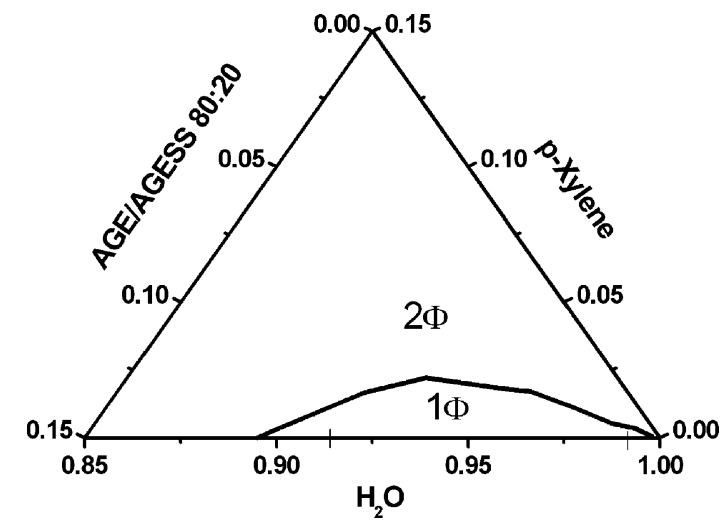

Figure 4. Phase diagram for the system AGE/AGESS 80:20/pxylene $/ \mathrm{H}_{2} \mathrm{O}$ in the water-rich region at $25^{\circ} \mathrm{C}$.

into the micelles). The $\mathrm{cmc}$ of this mixture in water was measured to establish the minimum amount of surfactant needed to obtain micelle formation. The cmc value obtained was $4.3 \times$ $10^{-3} \mathrm{w} / \mathrm{w} \%$.

Figure 4 shows that the amount of $p$-xylene solubilized in the 80:20 w/w AGE/AGESS micellar system is between 0.3 and $2 \%$ $\mathrm{w} / \mathrm{w}$ for $0.5-10 \% \mathrm{w} / \mathrm{w}$ surfactant concentration. It follows that the total organic phase fraction is comprised between 0.5 and $10 \% \mathrm{w} / \mathrm{w}$ reducing the total amount of the organic component of about $90-95 \%$.

Considering the nature of the oil phase and the very high interfacial area, typical of microemulsions, system A (Table 1) is expected to efficiently solubilize the acrylic copolymers. To confirm that, microemulsion A has been left in direct contact with a pure poly-EMA/MA pellet and, even in presence of large amounts of the polymer, no phase separation has been observed over a 6 month period. QELS experiments were performed on AGE/AGESS/ $p$-xylene/water system, before and after the addition of poly(EMA/MA).

Figure 5 A shows the weighted size distribution of the droplets' hydrodinamic diameter for microemulsion $\mathrm{A}$ as obtained by means of Laplace inversion of autocorrelation function through the CONTIN method. The distribution is centered at an average diameter value of $49 \mathrm{~nm}$ and is rather broad, as confirmed by the polydispersity value obtained from cumulant analysis (about $20 \%$ ). An excess of poly(EMA/MA) was added into the system and, after $36 \mathrm{~h}$, QELS measurements were performed on the solution. Light-scattering data point out an alteration of the relaxation time distribution (Figure 5B), indicating a change of droplet size. CONTIN analysis shows the formation of a second population, whose hydrodynamic diameter is peaked at $172 \mathrm{~nm}$ (Figure 5B) that can be attributed to oil droplets containing the solubilized polymer, cohexisting with "empty" microemulsion droplets (Figure 5B). This is also confirmed by double exponential and nonlinear least-squares (NNLS) analysis. ${ }^{35}$ This finding is somehow unexpected. To establish if the distribution is truly bimodal or if the system is approaching equilibrium, QELS measurements have been performed after 15 and 30 days from its preparation. CONTIN and cumulant analysis methods indicate an alteration of the diameter distribution with the formation of a very broad population at the equilibrium characterized by a mean diameter of $61.0 \mathrm{~nm}$ (Figure 5C). The size increase of the nanodroplets is the main reason of the increase of the viscosity value (from $0.97 \mathrm{mPa} \cdot \mathrm{s}$ for the pure microemulsion to $1.4 \mathrm{mPa} \cdot \mathrm{s}$ for the microemulsion in the presence of poly(EMA/MA) and after 1 month from the polymer addition to the microemulsion).

(35) Lawson, C. L.; Hanson, R. J. Solving Least Square Problems; Prentice Hall: Englewood Cliffs, NJ, 1974. 

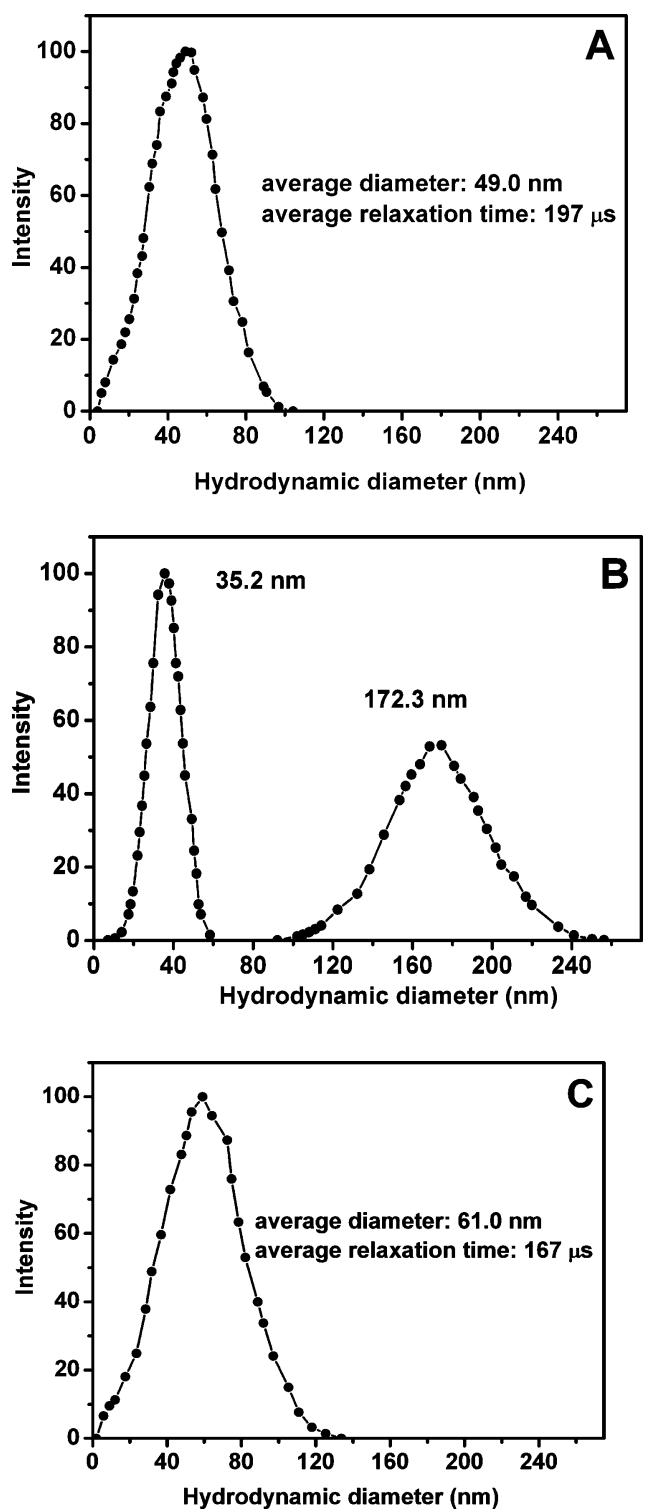

Figure 5. Intensity weighted size distributions of microemulsion droplets obtained from QELS measurements for microemulsion A before (A) and $36 \mathrm{~h}$ after (B) the solubilization of poly(EMA/MA). (C) Size distributions of microemulsion droplets for the nonaged poly(EMA/MA) enriched microemulsion at the equilibrium.

Both systems, before and after the addition of the copolymer, are characterized by a rheological Newtonian behavior (data not reported), suggesting that the microemulsion nanodroplets even after the solubilization of the copolymer can be still modeled as noninteracting rigid globular objects. ${ }^{36}$

It is well-known that the application of acrylic copolymer coatings onto a surface highly alters the physicochemical properties of the interface. ${ }^{37}$ This is very important in the case of paintings and is exemplified in Figure 6, where the wettability of both a glass and a mortar surface for the untreated surface, the surface with the paraloid coating, and the surface cleaned by using the microemulsion $\mathrm{A}$ are reported.

To verify the efficacy of the cleaning procedure using microemulsion A, contact angles were measured after the application of the microemulsion as described in the Experimental Section. A strong decrease of the contact angle to a value very

(36) Barnes, H. A.; Hutton, J. F.; Walters, K. An Introduction to Rheology; Elsevier: Amsterdam, 1989.

(37) Carretti, E.; Dei, L. Prog. Org. Coat. 2004, 49, 282.

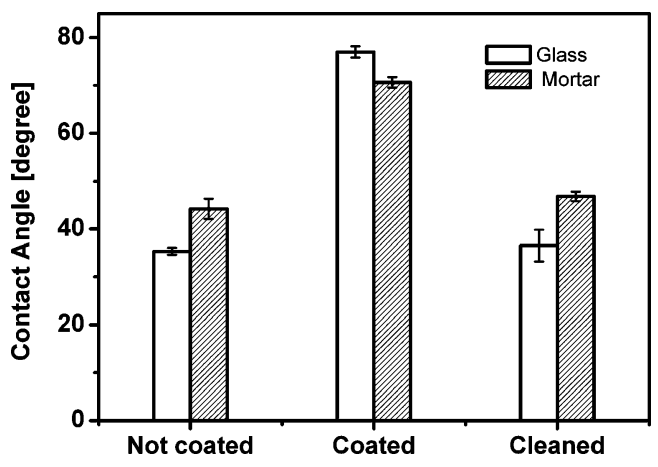

Figure 6. Contact angle behavior for water droplets on different solid surfaces. From left to right: "not coated" is the free solid surface, "coated" is the surface coated by a poly(EMA/MA) layer, and "cleaned" are the two coated surfaces after application of microemulsion A

close to that of the substrate before the poly(EMA/MA) application is observed. This clearly indicates the good "extraction" capacity of the microemulsion that completely removes the hydrophobic film from the surface of the substrates.

Gravimetric measurements further confirm that the polymer extraction from the surface is $>95 \%$ (w/w). The slightly different values of the contact angle investigated after the cleaning procedure, with respect to the nontreated supports, are due to the residual fraction of the acrylic copolymer $(<5 \%)$ still present on the surface. This very low residual amount of the polymer causes the modification of the three-phase contact line of the water drop onto the surface.

Microemulsion A efficacy as cleaning tool for the removal of aged acrylic polymers from painted surface was then tested on the a secco wall paints by Vecchietta in the Old Sacristy of Santa Maria della Scala in Siena, treated with PolyEMA/MA 70:30 at the end of the 1960s. Figure 7 shows a picture of the paint region where the cleaning test was carried out. The image in Figure 7B was collected at very low grazing angle from the same region where the cleaning test has been done; the glossy effect is clearly evident, indicating that the acrylic copolymer film was particularly thick, strongly modifying the optical properties of the paint surface. The image in Figure 7C was collected after the treatment with microemulsion $\mathrm{A}$ and subsequent washing with deionized water. Only in the cleaned region the glossy effect completely disappeared, indicating that microemulsion $\mathrm{A}$ is appropriate for naturally aged acrylic polymer extraction.

The quantitative determination of the efficacy of the microemulsion was obtained by FTIR spectra collected in the microreflectance mode from the surface of two microsamples taken from the same area of the paint, before and after the application of the microemulsion. The reflectance spectrum of the cleaned surface clearly shows that the acrylic layer had been successfully removed ( $>95 \%$ in a single application) because the signal at $1750 \mathrm{~cm}^{-1}$, because the $\mathrm{C}=\mathrm{O}$ stretching signal at $1750 \mathrm{~cm}^{-1}$, used as marker for the polymer, completely disappeared. ${ }^{1}$ To confirm this result, an FTIR analysis was also carried out on the residue of the extraction with trichloromethane made on the same microsample and no traces of organic materials were found, indicating the complete removal of the polymer layer during the cleaning of the painted surface.

The morphology of the surface has also been investigated by scanning electron microscopy on the same microsamples used for the FTIR. It is well-known that the main consequence of the deposition of a poly(EMA/MA) based coating is that, with respect to the untreated mortar, the surface appears "smoothed" and more homogeneous, with a partial occlusion of the pores with 


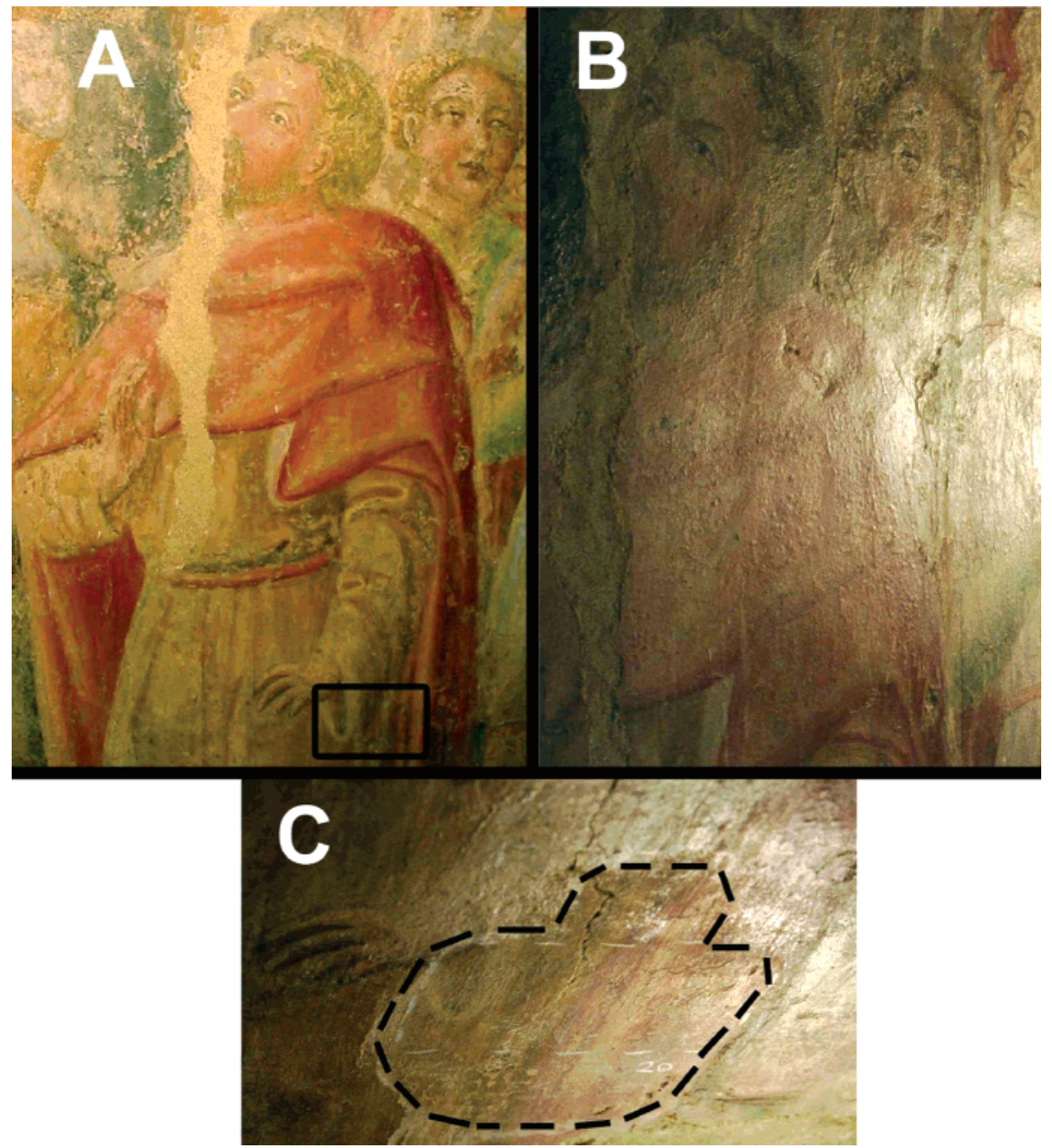

Figure 7. Santa Maria della Scala Sacristy, Siena, Italy. (A) Portion of the wall painting where copolymer solubilization tests by means of microemulsion A were carried out. (B) Grazing light image of the fresco. The glossy effect is due to the presence of the surface layer of poly(EMA/MA). (C) Grazing light image of the fresco region where the cleaning test was carried out after the applicaton of microemulsion A.
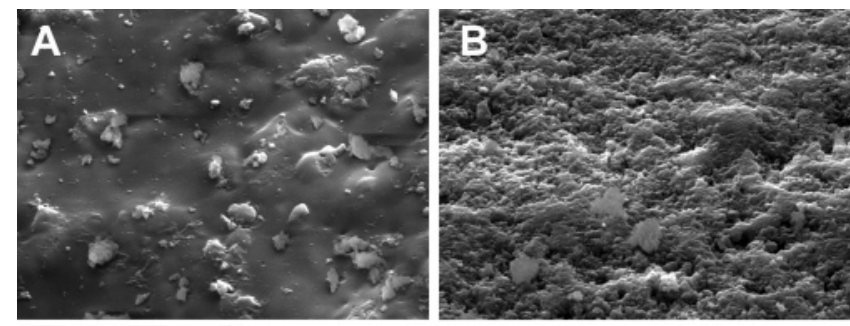

$20 \mu \mathrm{m}$

Figure 8. Vecchietta's fresco in Santa Maria della Scala, Siena, Italy. SEM micrograph of a microsample showing a thick layer of polymer before the treatment (A) and after (B) the application of microemulsion A.

diameter in the range of micrometers (Figure $8 \mathrm{~A}) .{ }^{37}$ After the microemulsion cleaning process, the morphology of the mortar surface is reverted to that of a noncoated mortar (Figure 8B). This result confirms the efficacy of microemulsion A as cleaning agents for painted surfaces. In particular, the use of microemulsions is particularly appropriate for the a secco paintings where the strength of the paint is fragile and easily detachable. The painted layer after a $2 \mathrm{~h}$ application of the microemulsion is completely clean and does not show any detachment or formation of craquelets, indicating that this method is, at the same time, effective in the removal of the surface polymer layer and, because of the very low oil content, completely harmless.
(2) Microemulsions for the Removal of Asphaltenes Crusts. Most of the problems caused by the flood of the Arno river to the Florence cultural heritage were related to the absorption (mediated by the porosity of the materials) of the substances dispersed or solubilized into the flooding water. Among them, a relevant problem was represented by hydrocarbons from fuels deposited inside the artifacts as asphaltenes, a mixture of hydrocarbons having complex structures of polyaromatic rings and short side chains. ${ }^{38}$ The presence of aged polymers combined with large amount of salts that usually form highly insoluble crusts, as for example the black crusts covering building facades because of vehicular traffic, is also a typical problem for most of the buildings in modern cities. The cleaning is often a very demanding and expensive process. In some cases, the removal of black crusts from the surfaces of historical and artistical interest (composed mainly by $\mathrm{CaSO}_{4} \cdot 2 \mathrm{H}_{2} \mathrm{O}$ and carbon particles) has been achieved by using an aqueous saturated solution of $\left(\mathrm{NH}_{4}\right)_{2}$ $\mathrm{CO}_{3}$. Unfortunately, this procedure is not helpful in the copresence of asphaltenes and sulfates. The application of ammoniun carbonate barely removes part of the sulfates while a fraction of asphaltenes could be removed using very aggressive and unsafe solvents as nitrobenzene and pyridine. ${ }^{39}$ One very simple and cheap way would be the application of microemulsion-based

(38) Yan Zhang, L.; Lawrence, S.; Xu, Z.; Masliyah, J. H. J. Colloid Interface Sci. 2003, 264, 128 .

(39) Da Silva Ramos, A. C.; Haraguchi, L.; Notrispe, F. R.; Loh, W.; Mohamed, R. S. J. Pet. Sci. Eng. 2001, 32, 201. 

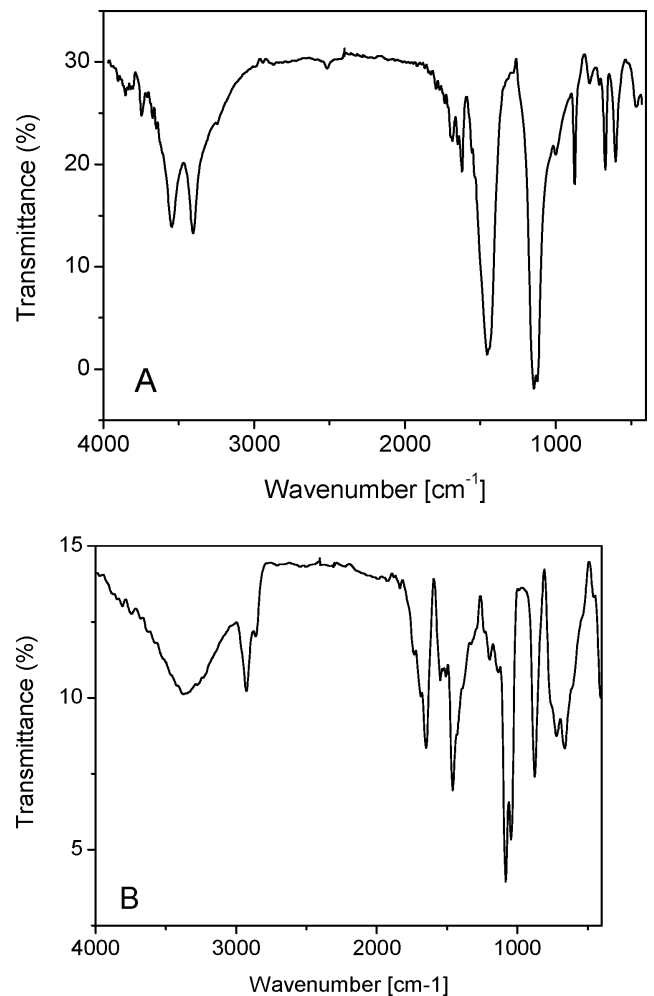

Figure 9. Oratorio di San Nicola al Ceppo, Florence, Italy. (A) FTIR spectrum from a microsample taken before the application of the microemulsion B. (B) FTIR spectrum collected on the residue of the extraction carried out by means of $\mathrm{CHCl}_{3}$, showing the complete removal of paraloid.

systems to specifically remove both salts and aged polymers in a single step.

We formulated a new microemulsion system composed of the nonionic Triton X-100 as a surfactant and $p$-xylene and $\left(\mathrm{NH}_{4}\right)_{2}$ $\mathrm{CO}_{3}$ solubilized in the microemulsion continuous phase. This new microemulsion merges two different restorations technologies: (1) detergency with an oil-in-water microemulsion and (2) desulfation according to the Ferroni-Dini method. ${ }^{40-42}$ The microemulsion was very efficient in removing in a single step both the aged black crusts composed by gypsum $\left(\mathrm{CaSO}_{4} \cdot 2 \mathrm{H}_{2} \mathrm{O}\right)$ and the degraded asphaltenes from the surface of the wall paintings in the Oratorio di San Nicola al Ceppo in Florence, Italy (17th century wall painting) (see Table 1 , microemulsion B), and constitutes a real breakthrough in conservation of Cutural Heritage and in detergency science.

The composition of the hard crusts from the Oratorio di San Nicola al Ceppo was qualitatively determined by FTIR analysis on a sample taken in the area where microemulsion B was applied (Figure 9A) and indicates that it is mainly composed of gypsum (the most important absorptions are at 3545, 3405, 3244, 1684, $1620,1144,1117,669$, and $604 \mathrm{~cm}^{-1}$, see Figure 9A). The presence of asphaltenes (absorptions at 2950, 2930, 2855, 1700, $1610,1460,1376$, and $1020-1049 \mathrm{~cm}^{-1}$ ) in the crusts was confirmed by a further FTIR investigation carried out by collecting a spectrum onto the extracted materials obtained dispersing the sample in $\mathrm{CHCl}_{3}$ for $36 \mathrm{~h}$ (Figure 9B).

(40) Ferroni, E.; Malaguzzi-Valerj, V.; Rovida, G. In Reports, Plenary Meeting, Amsterdam, Netherlands, 1969 - ICOM Committee for Conservation; The International Council of Museums: Paris, 1969.

(41) Ferroni, E.; Baglioni, P. In Proceedings of the symposium: Scientific methodologies applied to works of art, Florence, Italy, May 2-5, 1984; Parrini, P. L., Ed.; Montedison Progetto Cultura: Milan, Italy, 1986; pp 108-109.

(42) Ferroni, E. OPD Restauro 1999, 11, 97.

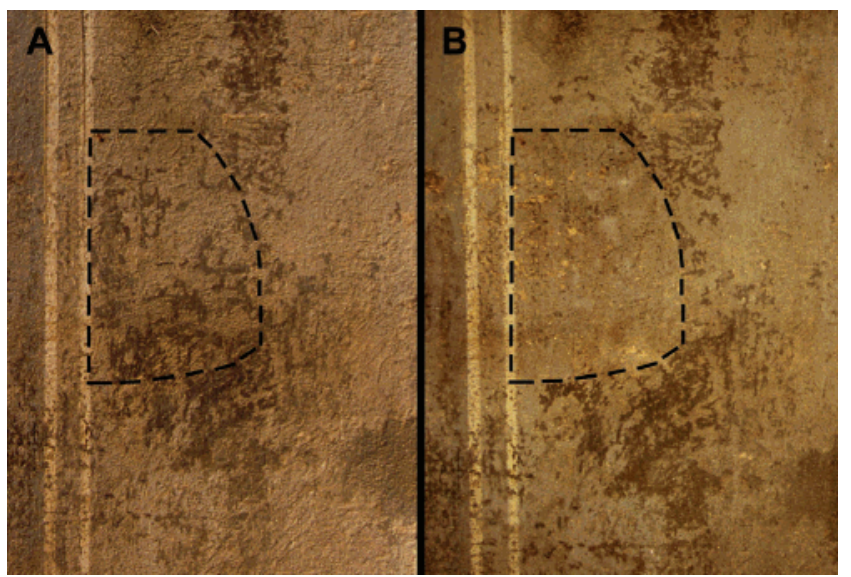

Figure 10. Oratorio di San Nicola al Ceppo, Florence, Italy. (A) Portion of the wall painting where solubilization tests of black crusts were carried out by means of microemulsion B before (A) and after (B) the application of the dispersed system.

APG based microemulsions could not be applied because the solubilization of $\left(\mathrm{NH}_{4}\right)_{2} \mathrm{CO}_{3}$ produced a phase separation in the microemulsion. A good alternative to APG was represented by the nonionic surfactant Triton $\mathrm{X}-100$. The addition of $\left(\mathrm{NH}_{4}\right)_{2}$ $\mathrm{CO}_{3}$ led to a decrease of the maximum $p$-xylene amount that can be loaded into the four-component-system (from $1.7 \%$ to $0.44 \%$ $(\mathrm{w} / \mathrm{w}))$. However, the $p$-xylene content was enough to achieve the optimal cleaning process. QELS measurements performed on this system indicated the formation of droplets characterized by a hydrodynamic radius of about $20.0 \mathrm{~nm}$.

Figure 10A reports an area contaminated by asphaltenes and gypsum black crusts where test were performed. Figure 10B was taken after the application of microemulsion B. Into the region indicated by the dotted line, the black crust completely disappeared indicating the complete removal of these deposits. FTIR analysis on microsamples taken from this area (see Figure 10B) did not show any signal typical of the crusts, confirming the complete removal of the organic and inorganic degraded materials.

Therefore, the excellent performance in the cleaning process (see Figure 10B) can be attributed to the synergistic action of the $p$-xylene nanodroplets combined with the $\left(\mathrm{NH}_{4}\right)_{2} \mathrm{CO}_{3}$ contained in the continuous aqueous phase.

\section{Conclusions}

The present study showed that acrylic polymers usually employed in works of art (or architecture) conservation and highly insoluble inorganic deposits containing organic materials can be solubilized in o/w microemulsions from nonionic surfactants, as alkyl polyglycoside or Triton X-100 both containing $p$-xylene as oil phase. The complete cleaning of an acrylic-contaminated painting can be successfully achieved by using an alkyl polyglycoside o/w microemulsions. This microemulsion system allows a consistent reduction (up to $95 \%$ ) of the total amount of the organic phase $(1 \% \mathrm{w} / \mathrm{w})$. The microemulsion is particularly efficient for the cleaning of a secco painting, a fragile painting difficult to clean with "conventional" methods as in the case of the restoration of the wall painting by Vecchietta in Santa Maria della Scala Sacristy, Siena, Italy. An additional microemulsion has been formulated for the specific and very demanding removal of salts and asphaltenes. This new system combines the microemulsion cleaning procedure with one of the most used methods (the Ferroni-Dini method) for sulfate removal from pictorial surfaces and represents the state of the art of the cleaning in the Cultural Heritage Conservation. The microemulsion show 
high efficacy and allows removing black crusts from works of art that are usually very difficult to remove.

The excellent results obtained in the "real cases" (wall paint by Vecchietta in Santa Maria della Scala Sagristy, Siena, Italy for APG based systems and Oratorio di San Nicola al Ceppo, Florence, Italy for the Triton X-100 microemulsions) suggested that these new o/w nanocompartimentalized systems can be adopted in the near future for conservation of cultural heritage, achieving, at the same time, the possibility of using a methodology with very low environmental impact and high performance for the cleaning of painted surfaces.

Acknowledgment. The authors thank the conservators Fabrizio Jacopini and Daniela Dini for their cooperation during restoration tests. Financial support from Consorzio Interuniversitario per lo Sviluppo dei Sistemi a Grande Interfase (CSGI) and MIUR is gratefully acknowledged.

LA700487S 